\title{
Direct prediction of nonlinear tearing mode saturation using a variational principle
}

\author{
J. Loizu ${ }^{1}$, Y.-M. Huang 2 , S. R. Hudson ${ }^{2}$, A. Baillod ${ }^{1}$, A. Kumar ${ }^{3}$, and Z. Qu ${ }^{3}$ \\ 1 École Polytechnique Fédérale de Lausanne, Swiss Plasma Center, CH-1015 Lausanne, Switzerland \\ 2 Princeton Plasma Physics Laboratory, PO Box 451, Princeton NJ 08543, USA \\ ${ }^{3}$ Mathematical Sciences Institute, the Australian National University, Canberra ACT 2601, Australia*
}

(Dated: June 8, 2020)

It is shown that the variational principle of multi-region relaxed magnetohydrodynamics (MRxMHD) can be used to predict the stability and nonlinear saturation of tearing modes in strong guide field configurations without resolving the dynamics and without explicit dependence on the plasma resistivity. While the magnetic helicity is not a good invariant for tearing modes, we show that the saturated tearing mode can be obtained as an MRxMHD state of a priori unknown helicity by appropriately constraining the current profile. The predicted saturated island width in a tearing-unstable force-free slab equilibrium is shown to reproduce the theoretical scaling at small values of $\Delta^{\prime}$ and the scaling obtained from resistive MHD simulations at large $\Delta^{\prime}$.

Magnetic reconnection is a ubiquitous phenomenon in the Universe that exhibits fast conversion of tremendous amounts of magnetic energy into kinetic energy. It is observed in solar eruptive flares [1,2] and in the interaction between the solar wind and the Earth's magnetic field [3]. Magnetic reconnection has an immediate practical importance. In magnetic fusion devices, reconnection drives fast relaxation processes, such as sawtooth-like temperature crashes in tokamaks [4], reversed field pinches [5], and stellarators [6], as well as other typically slower relaxation processes, such as neoclassical tearing modes in tokamaks [7], which degrade plasma confinement, slow plasma rotation, and can trigger plasma disruptions.

In the presence of a strong guide field (e.g., the toroidal field in tokamaks), reconnection often occurs via the tearing mode instability. Within resistive magnetohydrodynamics (MHD), tearing modes appear when a certain parameter, $\Delta^{\prime}$, which depends on the equilibrium profiles, exceeds a critical value, $\Delta_{c}$, which is zero except for toroidal effects $[8,9]$. A magnetic island then grows with a time scale between the ideal (Alfvén) time scale $\tau_{A}$ and the resistive (diffusion) time scale $\tau_{\eta}[10]$. In tokamaks, nonlinear saturation of the tearing mode is typically observed, and the corresponding saturated island width, $w_{\text {sat }}$, is a critical parameter for machine operation [7].

Numerical simulations of the growth and saturation of resistive tearing modes in realistic parameter regimes are expensive, primarily because of the very large Lundquist number, $S=\tau_{\eta} / \tau_{A} \gg 1$, which imposes resolving very disparate time scales [11]. For example, the ITER tokamak [12] can easily reach $S \sim 10^{9}$ in the core.

There are three crucial observations to make: (1) while the linear growth rate of the tearing mode always depends on resistivity, e.g. $\gamma \sim \eta^{3 / 5}$ in a cylinder [8], the instability parameter $\Delta^{\prime}$ does not, and the instability threshold is $\Delta_{c}=0$ except for toroidal corrections [9]; (2) while the nonlinear growth rate of the island depends on resistivity [13], the value of $w_{\text {sat }}$ does not [14]; and (3) the

*Electronic address: joaquim.loizu@epfl.ch very fact that a saturated state can be reached proves the existence of a nearby, generally three-dimensional MHD equilibrium. These observations raise the question: is there a variational principle that would allow direct calculation of the saturated tearing modes, without resolving the complex, resistivity-dependent dynamics? In this letter, we provide compelling analytical and numerical evidence that this is possible. This opens the possibility of performing much simpler numerical calculations, but most importantly provides deep insight into the fundamental physical mechanisms that underpin tearing mode formation and saturation, leading to new general understanding of magnetic reconnection phenomena.

The theory of Taylor relaxation [15, 16], which does not explicitly involve resistivity as a parameter, seems to indicate that it is indeed possible to find certain globally relaxed, saturated states by minimizing the classical MHD potential energy [17],

$$
W=\int_{V_{p}}\left(\frac{p}{\gamma-1}+\frac{B^{2}}{2 \mu_{0}}\right) d V
$$

where $V_{p}$ is the plasma volume, $p$ is the plasma pressure, and $\gamma$ is the adiabatic index. Taylor relaxed states satisfy a Beltrami equation, $\nabla \times \mathbf{B}=\mu \mathbf{B}$, with $\mu$ being a constant. They are obtained by taking $p=0$ in Eq. (1) and extremizing $W$ for arbitrary variations in the magnetic field subject to the only constraints of conserved total magnetic fluxes and conserved global magnetic helicity,

$$
K=\int_{V_{p}} \mathbf{A} \cdot \mathbf{B} d V,
$$

where $\mathbf{A}$ is the magnetic vector potential. In an infinitely conducting, ideal plasma, $K$ is an exact invariant in time, thereby reflecting the topological implications of Alfvén's theorem [18]. In a plasma with finite resistivity, $K$ is dissipated in time, but often much slower than $W$. In fact, it can be argued that $K$ is a good invariant during relaxation processes that involve small-scale magnetic fluctuations or fast reconnection time scales [16, 19]. Experiments have indeed confirmed the quasi-invariance of 
$K$ during sawtooth oscillations in tokamaks [20] and reversed field pinches [21]. Tearing modes, however, usually go through a slow nonlinear phase [13] and hence invariants other than $K$ shall be sought to describe their saturation in terms of a more general variational principle. Moreover, Taylor's theory cannot describe saturated states with non-trivial current profiles in strong guide field configurations. In fact, since the current density in a Taylor state satisfies $\mathbf{j}=\mu \mathbf{B}$, its spatial variation is quite limited in systems with a magnetic field whose magnitude, $B$, is almost uniform. In addition, Taylor states do not support pressure gradients, which are ubiquitous to any relevant magnetic fusion experiment.

Over a decade ago, the theory of multi-region relaxed MHD (MRxMHD) was proposed by Dewar et al [22, 23] in an attempt to generalize Taylor's relaxation theory and allow one to explore energetically favourable reconnection events while maintaining some ability to constrain the pressure and current profiles. MRxMHD starts with the same energy $W$, Eq. (1), but considers a constrained minimization with additional topological constraints. The plasma is partitioned into $N$ nested volumes separated by $N-1$ ideal interfaces that are assumed to remain magnetic surfaces during the minimization of the energy. In each volume, the plasma undergoes Taylor relaxation and the magnetic helicity, Eq. (2), is conserved within the volume, along with the toroidal and poloidal magnetic fluxes. While varying the plasma potential energy, $W$, the ideal interfaces are allowed to undergo geometrical deformations. The resulting MRxMHD equilibrium states satisfy

$$
\begin{aligned}
\nabla \times \mathbf{B}_{l} & =\mu_{l} \mathbf{B}_{l} \\
{\left[\left[p+\frac{B^{2}}{2 \mu_{0}}\right]\right]_{l} } & =0
\end{aligned}
$$

where $l=1, \ldots N$ labels the volumes, $\mu_{l}$ is a constant characterizing the Taylor states that is related to the parallel current, and $[[\cdot]]_{l}$ is the jump across the interface separating volumes $l$ and $l+1$.

A numerical solution to the MRxMHD equations can be found by using the Stepped-Pressure Equilibrium Code (SPEC), which can run in slab [24], cylindrical [25], or toroidal geometry [26]. Provided that the boundary geometry and the number $N$ of relaxation volumes are given, the solution is determined by a set of $N$ quadruplets, $\left\{p, \Psi_{t}, \Psi_{p}, K\right\}_{l=1 \ldots N}$. Namely, in each volume one must provide: the pressure $p$, the enclosed toroidal flux $\Psi_{t}$, the enclosed poloidal flux $\Psi_{p}$, and the enclosed magnetic helicity $K$. Alternatively, an equilibrium solution can be found by replacing, in each volume, the constraint on $K$ with a constraint on the Beltrami constant $\mu=\mathbf{j} \cdot \mathbf{B} / B^{2}$. This becomes particularly crucial if the current profile is to be constrained. In fact, while $K$ is not a good invariant for tearing modes, we will show that the quasi-invariance of the current profile can be exploited by SPEC to describe the saturated tearing equilibrium as an MRxMHD state of a priori unknown helicity.
We start by considering a force-free, doubly periodic, plasma current slab described by the following equilibrium magnetic field [27]: $\mathbf{B}=B_{z} \mathbf{e}_{z}+\mathbf{e}_{z} \times \nabla \psi$, where $z$ is the guide-field direction and the flux function $\psi(x)=$ $\psi_{0} / \cosh ^{2}(x)$ determines the "poloidal" magnetic field, $B_{y}(x)=\psi^{\prime}(x)$, as well as the "toroidal" current density $j_{z}(x)=\psi^{\prime \prime}(x)$ (Figure 1). The toroidal field, $B_{z}$, has a "radial" dependence such as to satisfy the force-balance condition $B_{z}^{2}(x)+B_{y}^{2}(x)=B_{0}^{2}$, where $B_{0}$ is the magnitude of the magnetic field on the resonant surface $(x=0)$. By setting $\psi_{0}=3 \sqrt{3} / 4$ we have that the maximum poloidal field is $B_{y, \max }=1$, and hence we can ensure that a strong guide field is present by choosing $B_{0} \gg 1$. This current sheet can be characterized by a radial equilibrium scale length $a \equiv \sqrt{\left|j_{z}(0) / j_{z}^{\prime \prime}(0)\right|}=1 / \sqrt{8} \approx 0.35$, and we choose a box size $L_{x}=2 \pi \gg a$. The poloidal extent is what controls the stability of the system and thus we choose $L_{y}=L_{z}=L$ as control parameter.

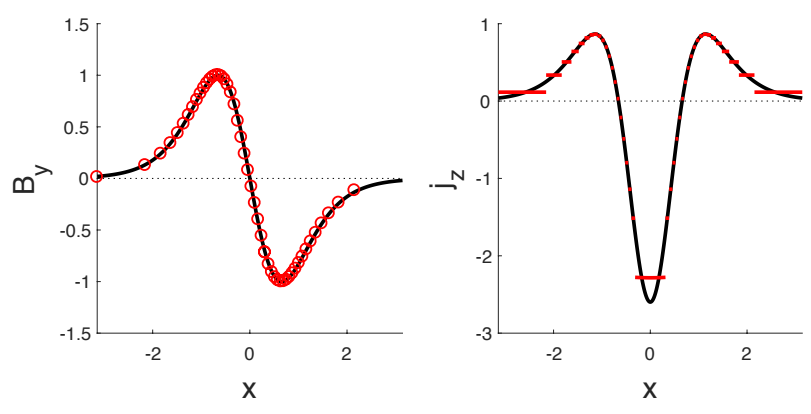

FIG. 1: Equilibrium radial profiles for the poloidal magnetic field, $B_{y}(x)=\psi^{\prime}(x)$, and the toroidal current density, $j_{z}(x)=$ $\psi^{\prime \prime}(x)$. Red circles and red stairs describe the equilibrium profiles obtained from the SPEC solution with $N=41, B_{0}=$ $10, L=2 \pi, \Psi_{w}=0.1$.

While this equilibrium is ideally stable [28], it is unstable to a tearing mode [29], which grows at the resonant surface $x=0$. The instability parameter for the tearing mode is independent of resistivity and can be calculated analytically [29],

$$
\Delta^{\prime}=\frac{2\left(5-k^{2}\right)\left(3+k^{2}\right)}{k^{2} \sqrt{4+k^{2}}}
$$

with an instability occurring for $\Delta^{\prime}>0$. Here $k$ is the poloidal wavenumber whose smallest possible value is $k=$ $2 \pi / L$. Hence $\Delta^{\prime}>0$ for $2 \pi / L<\sqrt{5}$. The condition for instability thus becomes a condition for the current sheet aspect ratio, $a / L<\sqrt{5 /\left(32 \pi^{2}\right)} \approx 0.126$ (Figure 2).

We now describe this current sheet equilibrium and its stability from the perspective of MRxMHD. In order to accurately approximate the equilibrium profiles, we construct an MRxMHD equilibrium with a large number of volumes $N \gg 1$. In each volume, we provide a quadruplet $\left\{p=0, \Psi_{t}, \Psi_{p}, \mu\right\}_{l=1 \ldots N}$ by proceeding as follows. First, we select the positions, $x_{l}$, of the interfaces defining the relaxation volumes, with $x_{0}=-L_{x} / 2=-\pi$ 


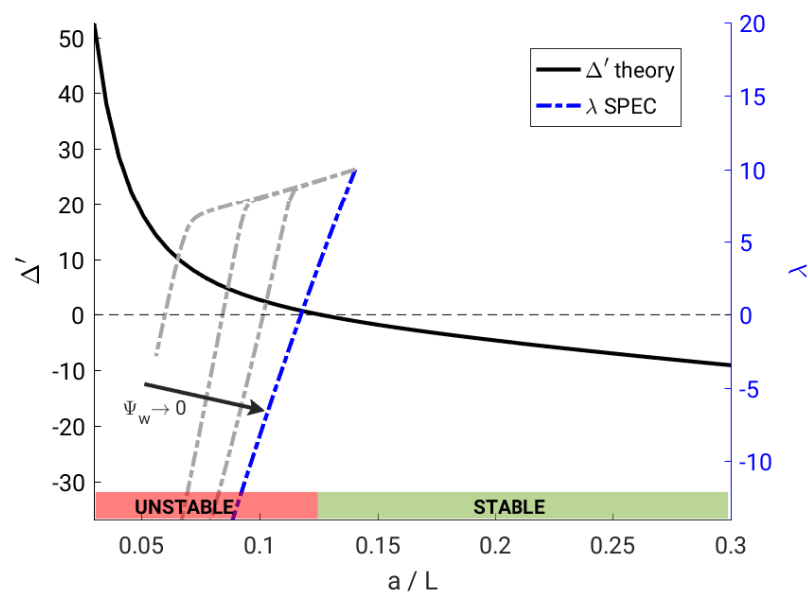

FIG. 2: Solid line: $\Delta^{\prime}$ versus $a / L$ obtained from Eq. (5) with $k=2 \pi / L$. Dashed lines: smallest eigenvalue $\lambda$ of $H$ computed from SPEC. Different curves are for different values of $\Psi_{w}$, the smallest being $\Psi_{w}=0.01$ (in blue). Here $N=121$.

and $x_{N}=L_{x} / 2=\pi$. In order to describe the equilibrium profiles efficiently we pack the interfaces where the gradients are larger, namely, by selecting interfaces with equal $\psi$-spacing instead of equal $x$-spacing. Second, we evaluate the enclosed poloidal and toroidal fluxes, $\Psi_{p}$ and $\Psi_{t}$, in each volume by using the analytical solution for $B_{y}(x)$ and $B_{z}(x)$. Finally, we evaluate the Beltrami constant by performing a radial average within each volume, $\mu=\left\langle\mathbf{j} \cdot \mathbf{B} / B^{2}\right\rangle_{x}$. For the special case of the volume containing the resonant surface, $x=0$, we allow for an arbitrary radial width that we parametrize by the enclosed toroidal flux normalized to the total toroidal flux, $0<\Psi_{w}<1$. In other words, the size of the resonant volume is proportional to $\Psi_{w}$. With this procedure, the SPEC equilibrium solution is only determined by the number of volumes, $N$, and the resonant flux $\Psi_{w}$. Figure 1 shows an example of profiles obtained from the SPEC solution for $N=41$ and $\Psi_{w}=0.1$. In the limit $N \rightarrow \infty$ and $\Psi_{w} \rightarrow 0$, the analytical profiles are retrieved exactly.

The stability of this MRxMHD equilibrium can be assessed by studying the derivative of the interfaces force balance, $f_{l}=\left[\left[p+B^{2} / 2 \mu_{0}\right]\right]_{l}$, with respect to poloidal perturbations in the interface geometry, $x_{l}$ [30]. Writing $f_{l}=\sum_{m} f_{l, m} \cos (m \theta)$ and $x_{l}=\sum_{m} x_{l, m} \cos (m \theta)$, where $\theta=2 \pi y / L$, we are interested in the matrix elements

$$
H_{i j}=\frac{\partial f_{l_{i}, m_{i}}}{\partial x_{l_{j}, m_{j}}}
$$

where $l_{q}=\lfloor 1+(q-1) / \mathcal{M}\rfloor$ and $m_{q}=q-1-\left(l_{q}-1\right) \mathcal{M}$, for $q=1 \ldots(N-1) \mathcal{M}$, with $N$ the number of relaxed volumes and $\mathcal{M}$ the number of Fourier modes. When $H$ is evaluated at fixed magnetic helicity and fluxes, its eigenvalues provide information about the stability of each eigenmode $m$ with the corresponding eigenfunction characterized by the eigenvector $\left\{x_{l, m}\right\}$.
The eigenvalues of $H$ are evaluated numerically using the SPEC code for different values of the equilibrium current sheet aspect ratio $a / L$. We observe that the smallest eigenvalue, referred to as $\lambda$, corresponds to an $m=1$ mode and becomes negative for sufficiently small $a / L$ (Figure 2). The value of $a / L$ at which $\lambda$ changes sign depends on the choice of $\Psi_{w}$ and, for $\Psi_{w} \rightarrow 0$, coincides with that at which $\Delta^{\prime}$ changes sign. Since $\lambda<0$ implies that the mode is unstable, we have just shown that the MRxMHD stability boundary is in agreement with linear tearing mode theory.

We now consider equilibria with $\Delta^{\prime}>0$, for which the tearing mode is expected to develop an island until saturation is reached. An exact nonlinear saturation theory, valid in the limit of small $\Delta^{\prime}$, was developed independently by Escande and Ottaviani [31] and by Militello and Porcelli [32]. In this theory, sometimes referred to as the POEM theory [27], the saturated island width is

$$
w_{\text {sat }}=2.44 \Delta^{\prime} a^{2}
$$

and thus linearly increases with $\Delta^{\prime}$ (Figure 3 ). We can also obtain the saturated island width at arbitrarily large values of $\Delta^{\prime}$ by solving, e.g., the compressible, viscoresistive, MHD equations in 2D [33],

$$
\begin{aligned}
\partial_{t} \rho+\nabla \cdot(\rho \mathbf{v}) & =0 \\
\partial_{t}(\rho \mathbf{v})+\nabla \cdot(\rho \mathbf{v} \mathbf{v}) & =-\nabla\left(\frac{B^{2}}{2}\right)-\nabla \cdot(\mathbf{B B})+\nu \nabla^{2}(\rho \mathbf{v}) \\
\partial_{t} \mathbf{B} & =\nabla \times\left[\mathbf{v} \times \mathbf{B}-\eta\left(\mathbf{j}-\mathbf{j}_{e q}\right)\right]
\end{aligned}
$$

where $\eta$ and $\nu$ are the resistivity and viscosity, respectively, and $\mathbf{j}_{e q}$ is the (initial) equilibrium current profile and represents a source term in Faraday's law that ensures the stationarity of the plasma current on resistive time-scales. As in the POEM theory, the toroidal current density at saturation is a flux-function,

$$
j_{z}=\left\langle j_{z, e q}\right\rangle_{\psi}
$$

where $\langle\cdot\rangle_{\psi}$ is the average over a flux-surface and $\psi(x, y)$ is the saturated poloidal flux function. Equation (9) implies the quasi-invariance of the current profile, the invariance being exact in the limit of small island width. This property will be exploited by SPEC to replace the invariance of $K$ with that of $j_{z}$ in the nonlinear calculations.

The saturated island width, $w_{\text {sat }}$, obtained by solving Eqs. (8) for different initial values of $\Delta^{\prime}$ is shown in Fig. 3. As reported by Loureiro et al [27], the predictions of the POEM theory are well recovered for a surprising range of values of $\Delta^{\prime} a$, up to about $\Delta^{\prime} a \approx 2$. For larger values of $\Delta^{\prime} a$, we also observe a curve-bending [27], with $w_{\text {sat }}$ below the POEM prediction. Importantly, the results become independent of resistivity for sufficiently small values of $\eta$ (and similarly for $\nu$ ), a property that can be anticipated by a mere rescaling of the velocity in Eqs. (8). More precisely, a transformation $\mathbf{v} \rightarrow \mathbf{v} / \eta$ leaves only dependencies of order $\eta^{2}$ and $\eta \nu$ when considering $\partial_{t}=0$. 


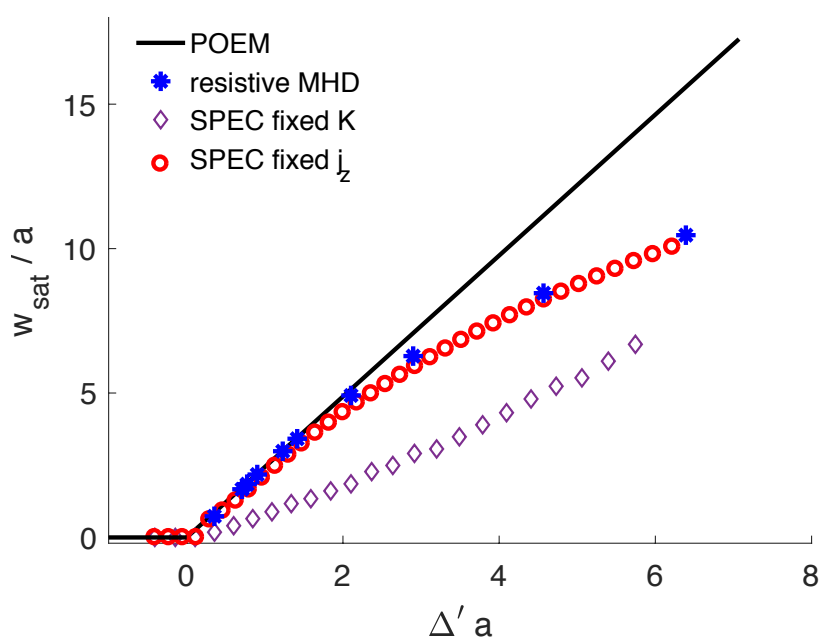

FIG. 3: Saturated island width, $w_{\text {sat }} / a$, as a function of $\Delta^{\prime} a$. Predictions shown for the POEM theory (solid line), the resistive MHD simulations solving Eqs. (8) with $\eta=10^{-2}$, $\nu=10^{-4}$, and conducting wall boundary conditions (blue stars), and the SPEC solution at fixed magnetic helicity (purple diamonds) and at fixed toroidal current (red circles).

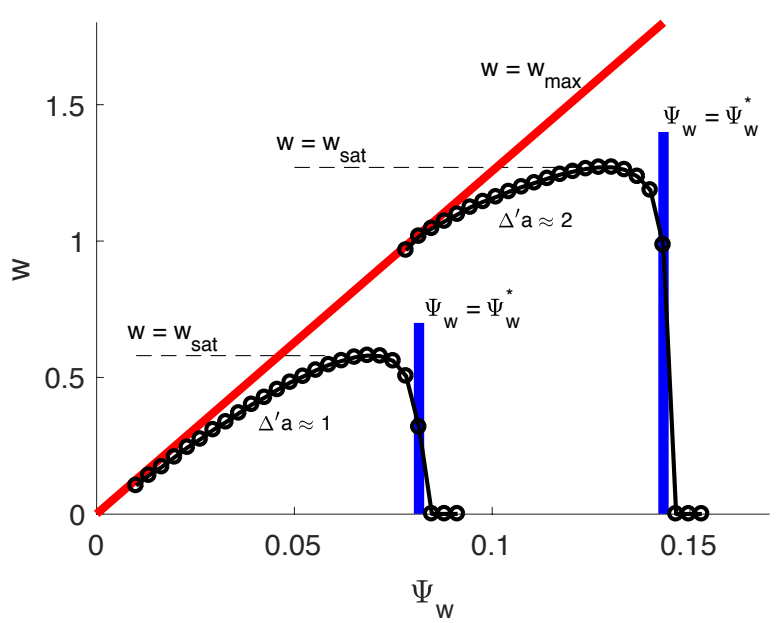

FIG. 4: Black circles: island width, $w$, obtained from SPEC as a function of $\Psi_{w}$, for two cases: $\Delta^{\prime} a \approx 1$ and $\Delta^{\prime} a \approx 2$. Vertical blue lines: location of the marginal point, $\Psi_{w}=\Psi_{w}^{*}$, above which the SPEC equilibrium is stable. Oblique red line: maximum allowed size of the island $w_{\max }=2 \Psi_{w} L_{x}=4 \pi \Psi_{w}$. Dashed lines: saturated island width obtained from SPEC, $w_{\text {sat }}$, as given by the maximum of the achieved island size.

SPEC is run nonlinearly in order to find saturated equilibria by starting from initially unstable equilibria, $\Delta^{\prime}>0$, and perturbing the geometry of the ideal interfaces, $x_{l}(\theta)$, according to the unstable eigenmode. For each value of $\Delta^{\prime}$, the current sheet aspect ratio $a / L$ is determined (Figure 2), and a choice must be made for $\Psi_{w}$. In the limit $\Psi_{w} \rightarrow 0$, the volume available for reconnection vanishes and therefore the maximum island width that is possibly achievable goes to zero. For increasingly large values of $\Psi_{w}$, the island is allowed to grow over a larger volume, although it may well only occupy a fraction of it. However, if the value of $\Psi_{w}$ exceeds a certain threshold, $\Psi_{w}^{*}$, the initial equilibrium becomes stable and no island grows at all. This is illustrated in Fig. 4 for two cases: one with $\Delta^{\prime} a \approx 1$ and one with $\Delta^{\prime} a \approx 2$. Obviously, for each $\Delta^{\prime}$, the saturated island width obtained with SPEC depends on $\Psi_{w}$. However, there is a distinct point, which we identify as $w_{\text {sat }}$, and that is the maximum achieved island width, which occurs at around $\Psi_{w} \approx \Psi_{w}^{*}$ (dashed lines in Fig. 4). We thus apply the following procedure: for each value of $\Delta^{\prime} a$, a linear stability analysis is first performed with SPEC and the value of $\Psi_{w}^{*}$ is obtained by solving $\lambda\left(\Psi_{w}\right)=0$. Then, SPEC is run nonlinearly with $\Psi_{w} \lesssim \Psi_{w}^{*}$ and the obtained value of $w_{\text {sat }}$ is extracted from the corresponding saturated state. It can also be easily verified that the result does not depend much on the exact choice of $\Psi_{w}$, i.e., that the corresponding value of $w$ is around its maximum (Figure 4).

The saturated island width, $w_{\text {sat }}$, obtained from SPEC nonlinear calculations for a wide range of $\Delta^{\prime} a$ is shown in Fig. 3 for two types of constraints. When the magnetic helicity and poloidal flux, $K$ and $\Psi_{p}$, in each volume are constrained to be exactly conserved (diamonds in Fig. 3), the value of $w_{\text {sat }}$ increases with $\Delta^{\prime} a$ but stays below the predictions of both POEM and resistive MHD simulations. This discrepancy reflects the fact that the magnetic helicity is not well conserved during the slowly reconnecting tearing mode [19]. However, when the toroidal current in each volume is constrained to be fixed (circles in Fig. 3), the scaling of $w_{\text {sat }}$ with $\Delta^{\prime} a$ is well reproduced for the entire range of $\Delta^{\prime} a$. The constraint on the current in SPEC is in fact an approximation of Eq. (9) and can be achieved by following the approach described in Ref. [34], i.e. by constraining $\mu$ to the initial value and iterating on $\Psi_{p}$ in each volume in order to avoid the formation of sheet currents on the interfaces, thereby ensuring that the current profile remains smooth. We would like to emphasize that, regardless of the applied constraint and for all $\Delta^{\prime}$, the obtained equilibria (1) have lower energy than the initial state, (2) satisfy the MRxMHD equilibrium equations, and (3) correspond to minima of the MRxMHD energy functional [26]. It is also important to notice a limitation of our results. For sufficiently large islands, $w / a \gg\left(\kappa_{\perp} / \kappa_{\|}\right)^{1 / 4}$, the flattening of the temperature modifies the plasma resistivity and thus $j_{z}(\psi)$ must be modified accordingly in order to balance the constant induced electric field [35]. Here $\kappa_{\|}$and $\kappa_{\perp}$ are the parallel and perpendicular heat conductivities. The prediction of $w_{\text {sat }}$ with SPEC in such regime [35] is beyond the scope of this paper and is left for future investigations. In general, our procedure should be able to directly predict the saturation of tearing modes so as long as a constraint akin to Eq. (9) is obtained from the considered model equations.

We would like to remark that the SPEC results shown 
in Fig. 3 are numerically converged in the sense that increasing further the Fourier resolution (here $m=4$ modes are considered) or the number of volumes (here $N=121$ ) does not significantly change the values of $w_{\text {sat }}$. Finally, we would like to emphasize that each resistive MHD calculation (each blue star in Fig. 3) takes about $1 \mathrm{CPU}$ hour, while each corresponding SPEC run (red circles in Fig. 3) takes about 5 seconds.

In this letter, we have demonstrated, for the first time, that it is possible to use an equilibrium code to directly predict the nonlinear saturation of tearing modes, without resolving the complex resistive-dependent dynamics and without free parameters. These saturated states are lower energy MRxMHD equilibria of a priori unknown helicity and are obtained by exploiting the quasiinvariance of the current profile. These findings provide a precise answer to the recent suggestion that tearing saturation occurs "when reaching some topologically constrained minimal energy state" [14]. In practice, numerical codes like SPEC or BIEST [36], which calculate MRxMHD equilibria in toroidal geometry, may be used to quickly predict saturated island widths in toka- maks and arbitrarily shaped stellarators. To that aim, future investigations will start by extending these calculations to cylindrical geometry, the results of which can be verified against generalizations of the POEM theory to asymmetric equilibrium current profiles [37].

We acknowledge very helpful discussions with Amitava Bhattacharjee, Per Helander, Robert Dewar, Adelle Wright, Alessandro Zocco and Jonathan Graves. This work has been carried out within the framework of the EUROfusion Consortium and has received funding from the Euratom research and training programme 2014 2018 and 2019 - 2020 under grant agreement No 633053. The views and opinions expressed herein do not necessarily reflect those of the European Commission. This work was also supported by a grant from the Simons Foundation/SFARI (560651, AB). The data that support the findings of this study are available from the corresponding author upon reasonable request.
[1] Kazunari Shibata and Tetsuya Magara. Solar Flares : Magnetohydrodynamic Processes. Living Reviews of Solar Physics, 8(6), 2011.

[2] Satoshi Inoue, Kanya Kusano, Jörg Büchner, and Jan Skála. Formation and dynamics of a solar eruptive flux tube. Nature Communications, 9:1-11, 2018.

[3] Thomas Earle Moore, James L Burch, and Roy B Torbert. Magnetic reconnection. Nature Physics, 11(August):611-613, 2015.

[4] R. J. Hastie. Sawtooth Instability in Tokamak Plasmas. Astrophysics and Space Science, 256(1-2):177-204, 1997.

[5] B E Chapman, A F Almagri, M Cekic, D. J. Den Hartog, S C Prager, and J S Sarff. Sawteeth and energy confinement in the Madison Symmetric Torus reversedfield pinch. Physics of Plasmas, 3(3):709-711, mar 1996.

[6] R. C. Wolf, A. Alonso, S. Äkäslompolo, J. Baldzuhn, M. Beurskens, C. D. Beidler, C. Biedermann, H.-S. Bosch, S. Bozhenkov, R. Brakel, H. Braune, S. Brezinsek, K.-J. Brunner, H. Damm, A. Dinklage, P. Drewelow, F. Effenberg, Y. Feng, O. Ford, G. Fuchert, Y. Gao, J. Geiger, O. Grulke, N. Harder, D. Hartmann, P. Helander, B. Heinemann, M. Hirsch, U. Höfel, C. Hopf, K. Ida, M. Isobe, M. W. Jakubowski, Y. O. Kazakov, C. Killer, T. Klinger, J. Knauer, R. König, M. Krychowiak, A. Langenberg, H. P. Laqua, S. Lazerson, P. McNeely, S. Marsen, N. Marushchenko, R. Nocentini, K. Ogawa, G. Orozco, M. Osakabe, M. Otte, N. Pablant, E. Pasch, A. Pavone, M. Porkolab, A. Puig Sitjes, K. Rahbarnia, R. Riedl, N. Rust, E. Scott, J. Schilling, R. Schroeder, T. Stange, A. von Stechow, E. Strumberger, T. Sunn Pedersen, J. Svensson, H. Thomson, Y. Turkin, L. Vano, T. Wauters, G. Wurden, M. Yoshinuma, M. Zanini, and D. Zhang. Performance of Wendelstein 7-X stellarator plasmas during the first divertor operation phase. Physics of Plasmas,
26(8):082504, 2019.

[7] R. J. La Haye. Neoclassical tearing modes and their control. Physics of Plasmas, 13(5):055501, may 2006.

[8] Roscoe B White. The Theory of Toroidally Confined Plasmas. IMPERIAL COLLEGE PRESS, jan 2014.

[9] A. H Glasser, J. M Greene, and J. L Johnson. Resistive instabilities in general toroidal plasma configurations. Physics of Fluids, 18(7):875, 1975.

[10] R J Goldston and $\mathrm{P} \mathrm{H}$ Rutherford. Introduction to Plasma Physics. Institute of Physics Publishing, 1995.

[11] Hinrich Lütjens and Jean-François Luciani. The XTOR code for nonlinear 3D simulations of MHD instabilities in tokamak plasmas. Journal of Computational Physics, 227(14):6944-6966, jul 2008.

[12] T.C Hender, J.C Wesley, J. Bialek, A. Bondeson, A.H Boozer, R.J Buttery, A. Garofalo, T.P Goodman, R.S Granetz, Y. Gribov, O. Gruber, M. Gryaznevich, G. Giruzzi, S. Günter, N. Hayashi, P. Helander, C.C Hegna, D.F Howell, D.A Humphreys, G.T.A Huysmans, A.W Hyatt, A. Isayama, S.C Jardin, Y. Kawano, A. Kellman, C. Kessel, H.R Koslowski, R.J. La Haye, E. Lazzaro, Y.Q Liu, V. Lukash, J. Manickam, S. Medvedev, V. Mertens, S.V Mirnov, Y. Nakamura, G. Navratil, M. Okabayashi, T. Ozeki, R. Paccagnella, G. Pautasso, F. Porcelli, V.D Pustovitov, V. Riccardo, M. Sato, O. Sauter, M.J Schaffer, M. Shimada, P. Sonato, E.J Strait, M. Sugihara, M. Takechi, A.D Turnbull, E. Westerhof, D.G Whyte, R. Yoshino, H. Zohm, Disruption Group, the ITPA MHD, and Magnet. Chapter 3: MHD stability, operational limits and disruptions. Nuclear Fusion, 47(6):S128-S202, jun 2007.

[13] P. H. Rutherford. Nonlinear growth of the tearing mode. Physics of Fluids (1958-1988), 16(11):1903-1908, 1973.

[14] A. Poyé, O. Agullo, A. Smolyakov, S. Benkadda, and $\mathrm{X}$. Garbet. Dynamics of magnetic islands in large $\Delta$ 
regimes. Physics of Plasmas, 21(2), 2014.

[15] J. B. Taylor. Relaxation of toroidal plasma and generation of reverse magnetic fields. Physical Review Letters, 33(19):1139-1141, 1974.

[16] J. B. Taylor. Relaxation and magnetic reconnection in plasmas. Reviews of Modern Physics, 58(3):741-763, 1986.

[17] M. Kruskal and R. Kulsrud. Equilibrium of a magnetically confined plasma in a toroid. Journal of Nuclear Energy (1954), 7(3-4):297, 1958.

[18] J P Freidberg. ideal MHD. Cambridge University Press, 2014.

[19] Mitchell A Berger. Introduction to magnetic helicity. Plasma Physics and Controlled Fusion, 41:166-176, 1999.

[20] W.W. Heidbrink and T.H. Dang. Magnetic helicity is conserved at a tokamak sawtooth crash. Plasma Physics and Controlled Fusion, 42:L31-L36, 2000.

[21] H. Ji, S. C. Prager, and J. S. Sarff. Conservation of Magnetic Helicity during Plasma Relaxation. Physical Review Letters, 74(15):2945-2948, apr 1995.

[22] M. J. Hole, S. R. Hudson, and R. L. Dewar. Stepped pressure profile equilibria in cylindrical plasmas via partial Taylor relaxation. Journal of Plasma Physics, $72(06): 1167,2006$.

[23] M.J Hole, S.R Hudson, and R.L Dewar. Equilibria and stability in partially relaxed plasmavacuum systems. $\mathrm{Nu}$ clear Fusion, 47(8):746-753, 2007.

[24] J. Loizu, S. Hudson, A. Bhattacharjee, and P. Helander. Magnetic islands and singular currents at rational surfaces in three-dimensional magnetohydrodynamic equilibria. Physics of Plasmas, 22(2), 2015.

[25] J. Loizu, S.R. Hudson, and C. Nührenberg. Verification of the SPEC code in stellarator geometries. Physics of Plasmas, 23(11), 2016.

[26] S. R. Hudson, R. L. Dewar, G. Dennis, M. J. Hole, M. McGann, G. Von Nessi, and S. Lazerson. Computation of multi-region relaxed magnetohydrodynamic equilibria. Physics of Plasmas, 19(11), 2012.

[27] N F Loureiro, S C Cowley, W D Dorland, M G Haines, and A A Schekochihin. X -Point Collapse and Saturation in the Nonlinear Tearing Mode Reconnection. Physical Review Letters, 95, 2005.

[28] J P Goedbloed and R Y Dagazian. Kinks and tearing modes in simple configurations. Physical Review A, 4(4):1554-1560, 1971.

[29] F Porcelli, D Borgogno, F Califano, D Grasso, M Ottaviani, and $\mathrm{F}$ Pegoraro. Recent advances in collisionless magnetic reconnection. Plasma Physics and Controlled Fusion, 44:B389-B405, 2002.

[30] J Loizu and S R Hudson. Multi-region relaxed magnetohydrodynamic stability of a current sheet. Physics of Plasmas, 26, 2019.

[31] D F Escande and M Ottaviani. Simple and rigorous solution for the nonlinear tearing mode. Physics Letters, Section A: General, Atomic and Solid State Physics, 323:278-284, 2004

[32] F Militello and F Porcelli. Simple analysis of the nonlinear saturation of the tearing mode. Physics of Plasmas, $11,2004$.

[33] Yi-min Huang and A Bhattacharjee. Turbulent magnetohydrodynamic reconnection mediated by the plasmoid instability. The Astrophysical Journal, 818(20), 2016.

[34] J. Loizu, S. R. Hudson, C. Nührenberg, J. Geiger, and P. Helander. Equilibrium beta-limits in classical stellarators. Journal of Plasma Physics, 83(6), 2017.

[35] R. J. Hastie, F. Militello, and F. Porcelli. Nonlinear saturation of tearing mode Islands. Physical Review Letters, 95(6):5-8, 2005.

[36] Dhairya Malhotra, Antoine Cerfon, Lise Marie ImbertGérard, and Michael O'Neil. Taylor states in stellarators: A fast high-order boundary integral solver. Journal of Computational Physics, 397:108791, 2019.

[37] N. Arcis, D. F. Escande, and M. Ottaviani. Rigorous approach to the nonlinear saturation of the tearing mode in cylindrical and slab geometry. Physics of Plasmas, 13(5):052305, may 2006. 